\title{
La BnF au défi de la numérisation des objets
}

\section{Virginie Louis, Dominique Maillet et Olivier Piffault}

\section{(2) OpenEdition}

Journals

Édition électronique

URL : http://journals.openedition.org/rbnu/597

DOI : 10.4000/rbnu.597

ISSN : 2679-6104

\section{Éditeur}

Bibliothèque nationale et universitaire de Strasbourg

\section{Édition imprimée}

Date de publication : 1 novembre 2018

Pagination : 20-25

ISBN : 9782859230784

ISSN : 2109-2761

\section{Référence électronique}

Virginie Louis, Dominique Maillet et Olivier Piffault, «La BnF au défı de la numérisation des objets », La Revue de la BNU [En ligne], 18 | 2018, mis en ligne le 01 juillet 2019, consulté le 13 décembre 2020. URL : http://journals.openedition.org/rbnu/597 ; DOI : https://doi.org/10.4000/rbnu.597

le la BNU est mise à disposition selon les termes de la Licence Creative Commons Attribution - Pas d'Utilisation Commerciale - Partage dans les Mêmes Conditions 4.0 International. 

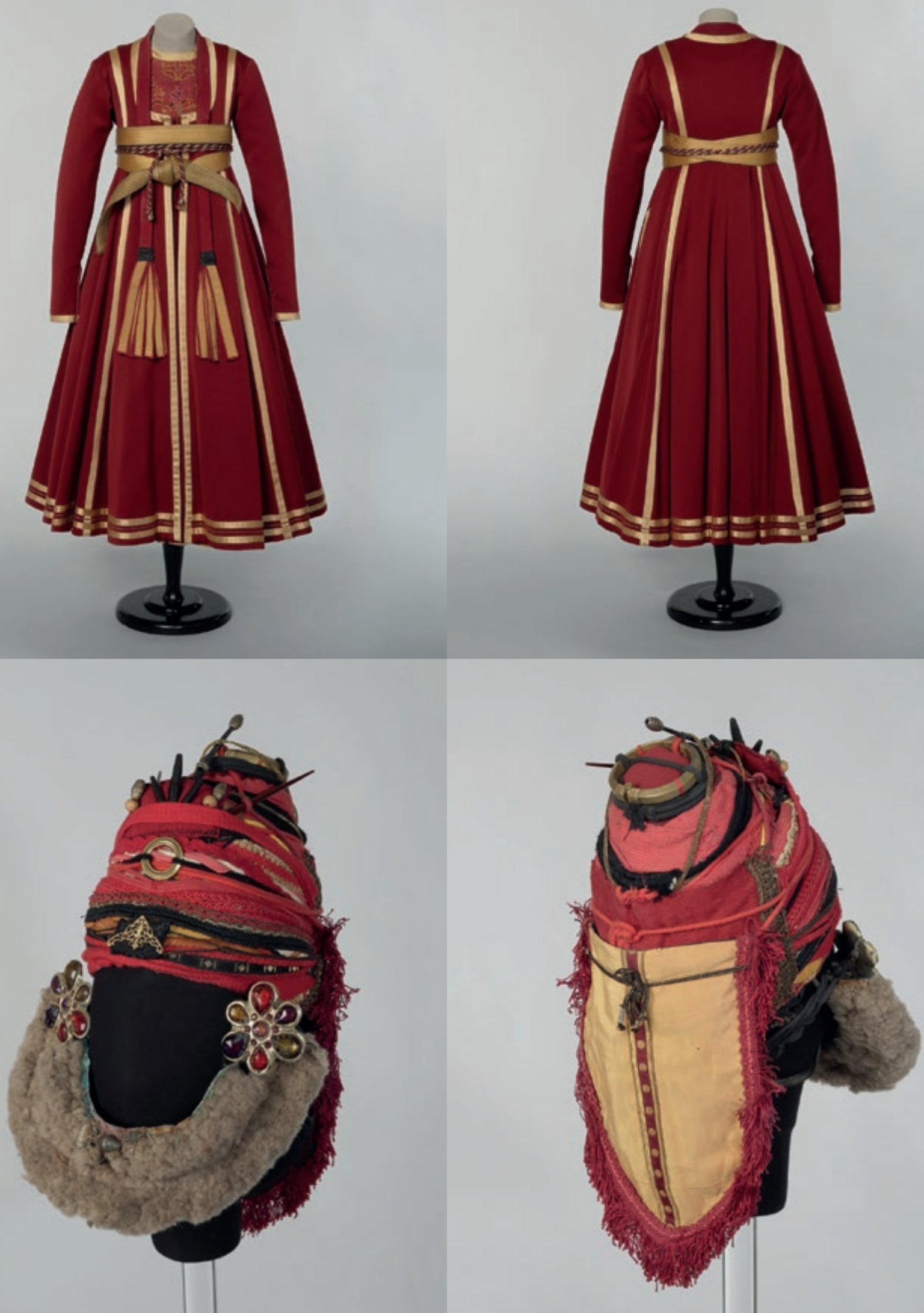

Costume du Théâtre du Soleil : rôle d'un coryphée interprété par Nirupama Nityanandan dans Agamemnon d'Eschyle (1990; coll. BnF) 


\section{LA BNF AU DÉFI DE LA NUMÉRISATION DES OBJETS \\ PAR VIRGINIE LOUIS, DOMINIQUE MAILLET ET OLIVIER PIFFAULT}

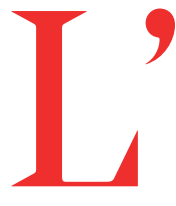

accès sans limites de lieux, d'horaires et de statuts de lecteurs aux documents est « l'objectif le plus ambitieux [...] le pari le plus difficile » du programme fixé en 1990 dans « Bibliothèque de France, le projet, les rapports des groupes de travail », introduisant une révolution tant documentaire que citoyenne par « la généralisation de l'accès au patrimoine " au moyen de la numérisation. Pensée autour des livres, puis de la presse, cette ambition a été reprise dans le contexte du bouleversement du web par tous les musées nationaux, l'Inventaire des monuments historiques et la plupart des institutions culturelles. Ayant consacré la bibliothèque numérique Gallica aux supports de l'écrit, la BnF n'a pas souhaité créer une iconothèque particulière pour ses collections d'objets, dont la variété et l'ampleur renvoient autant aux anciens trésors royaux qu'aux cabinets de curiosités, collections naturalistes, scientifiques ou ethnographiques. Le choix d'enrichir le contenu de Gallica avec les "non livres » a donc soulevé de multiples questionnements sur les modalités de numérisation « en volume », sur le traitement de ces données, leur présentation et leur valorisation.
S'agit-il d'une numérisation " sèche " de sauvegarde, ou de vues " esthétisantes " ? Que signifie une couverture exhaustive d'un objet ? Comment rendre la matérialité des œuvres et suivre les états évolutifs liés à la restauration, au nettoyage, à l'exposition même ? Quelle est la demande des usagers, celle des conservateurs ? Quelles technologies répondent le mieux à ces enjeux ? Les réponses sont aussi complexes, variées et évolutives que les fonds sont composites, et nous présentons ici la façon dont s'opère depuis dix ans le compromis entre ces tensions.

La filière de prises de vues d'objets a été initialement créée pour les 2000 maquettes de décors en volumes de la Bibliothèque-musée de l'Opéra. La compréhension du décor de ces maquettes ne peut se faire qu'une fois l'ensemble monté. Leur numérisation systématique semblait évidente pour permettre, au-delà de l'exposition, d'en garder et d'en diffuser une trace virtuelle. 2007-2008 ont été les années-test pour mettre en place l'organisation nécessaire (choix du matériel, des équipes et établissement des protocoles de prises de vues). Depuis 2009, des sessions sont ainsi programmées annuellement. Mais la BnF possède une bien plus large collection d'objets, et d'autres dépar- 
tements se sont inscrits dans ce processus qui permet une diffusion inédite, préservant les originaux. Citons celui des Monnaies, médailles et antiques (pour sa collection de monnaies et pour ses sceaux, intailles, camées, bijoux, sculptures ou statuettes), mais aussi celui des Arts du spectacle (marionnettes, masques, costumes), ou encore ceux des Cartes et plans (globes), des Estampes (moules de cartes à jouer, bois gravés), ou enfin de l'Audiovisuel avec sa prestigieuse collection Charles Cros (voir sur ces différents sujets l'article de Denis Bruckmann p. 9).

\section{MÉTHODOLOGIE DE CHANTIER : L'INCONTOURNABLE PROTOCOLE}

Au-delà d'une cohérence dans la présentation au public d'une collection d'objets numérisés, les protocoles sont l'outil de dialogue entre le conservateur responsable $\mathrm{du}$ fonds à photographier et l'équipe de photographes. Chacun d'entre eux est soumis, avant sa mise en œuvre, à la validation de l'équipe chargée des collections. Cette documentation précise permet également de faire intervenir des photographes différents, sans que chacun ne se repose la question de la façon de s'y prendre ni ne rajoute une subjectivité artistique personnelle.

Pour chaque type d'objets, le protocole indique la succession des vues à réaliser. Nous nous rapprochons de la numérisation de l'écrit pour laquelle nous recherchons la fidélité à l'original, et non pas sa valorisation. Nous avons en tête également la notion de couverture complète. Il en est de même pour les prises de vues d'objets : le but n'est pas d'obtenir une image flatteuse, mais une couverture photographique complète de l'objet non mis en scène, qui remplace une observation réelle de l'original. D'où la nécessité, selon les cas, de «faire le tour précis de l'objet» et de bannir les éclairages en clair-obscur. Une seule « photo de couverture " de l'objet pris dans son ensemble le met en scène de façon plus artistique.

Chaque nouvelle collection engendre son protocole, préalable indispensable à la photographie. Et tout objet, quel qu'il soit, a son propre mode de lecture. Il n'y a donc pas une numérisation, mais des numérisations d'objets.

\section{RENDRE L'ÉPHÉMÈRE ET L'INVISIBLE}

\section{Monnaies et camées, une approche classique}

Les monnaies sont les seuls objets de la BnF traités par un prestataire installé sur site. Notre collection grecque a été numérisée dans sa totalité, suivie par celle des jetons, des monnaies byzantines, romaines et françaises. Celles-ci sont en bronze, or, laiton, argent mat, argent brillant ou cuivre, avec des reliefs et des états divers. Les trois principales difficultés sont les suivantes : restituer la brillance des monnaies en or et en argent sans générer des reflets parasitant la lecture des droits et revers ; obtenir une netteté uniforme sur des objets en relief aux formes asymétriques ; enfin placer correctement la monnaie, alignée sur son sens de lecture.

\section{Les maquettes en volume, une mise en scène fugace}

Les maquettes de décors d'opéra sont restaurées, puis temporairement montées en volume pour exposition. Nous nous trouvons simples spectateurs ou acteurs à des positionnements différents, avec un œil et un point de vue complémentaires sur ce décor : assis au fond du théâtre, à la corbeille, à l'orchestre, placés au milieu, à droite ou à gauche dans la salle, laissant la place à l'acteur assis au bord de la scène puis nous promenant dans les différentes strates du décor... C'est cette idée qui nous a guidés pour la création du protocole : les objectifs et les focales des appareils photo changent pour restituer ces différents points de vue, et l'ensemble permet au lecteur internaute de voir ce décor et de s'y promener de façon certainement beaucoup plus complète que s'il avait la maquette face à lui. Les lumières plates à caractère documentaire laissent la place à des lumières chaudes évoquant les éclairages des becs de gaz de la scénographie du siècle dernier, complétant ainsi la lisibilité du spectacle. 

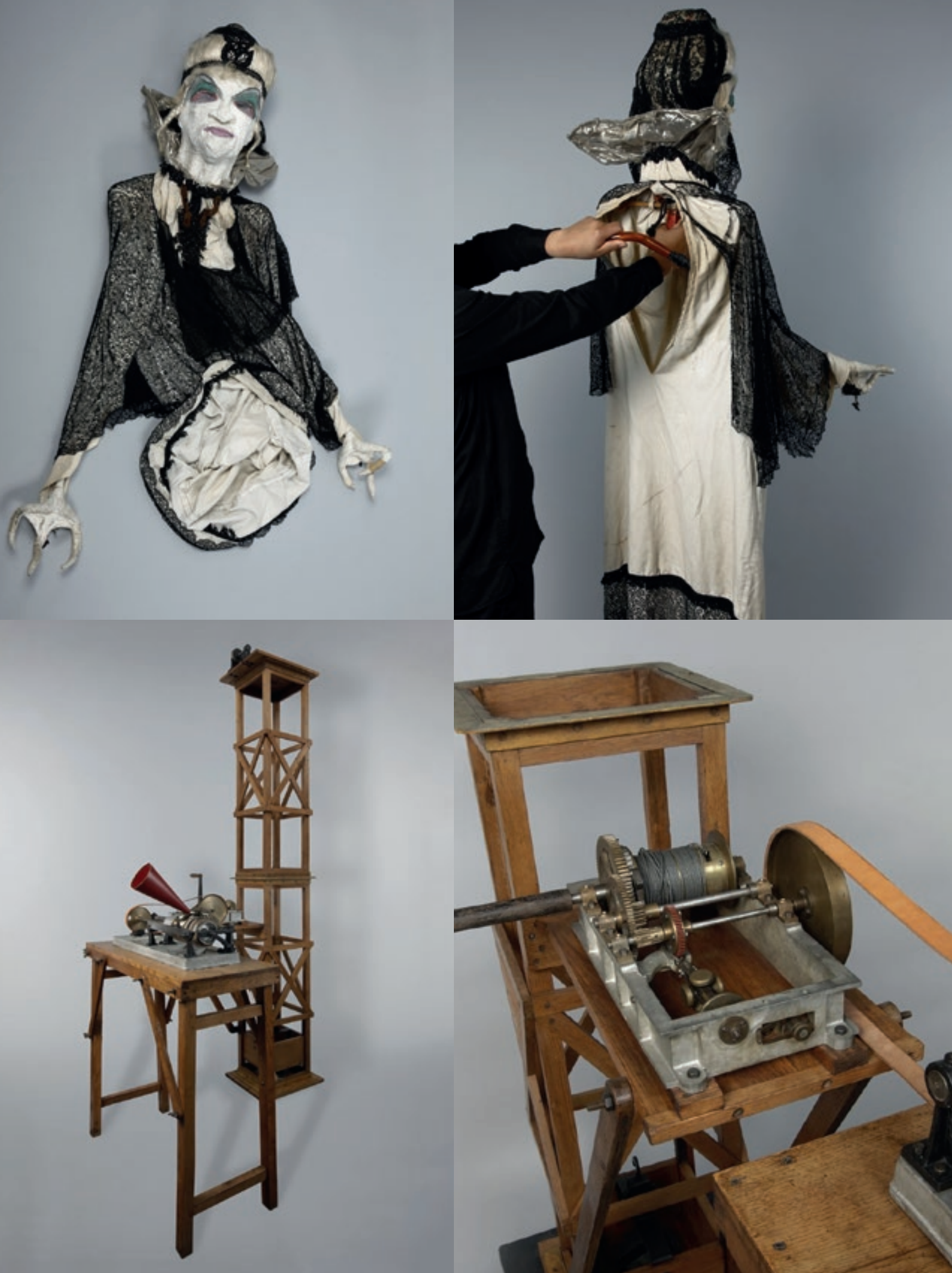

(En haut) Marionnette, fonds Dominique Houdart - Jeanne Heuclin. II. Activités théâtrales de la Compagnie Dominique Houdart - Jeanne Heuclin. A. Spectacles créés par la compagnie. Arlequin poli par l'amour (1972; Houdart) : marionnettes. La fée (1964-2010 ; coll. BnF)

(En bas) Collection Charles Cros : phonographes à contrepoids pour enregistrement professionnel.

Pathé : phonographe à contrepoids ou « puits à pétrole » des Archives de la parole (date d'édition : 1911-1928; coll. BnF) 


\section{Derrière le masque}

Le département des Arts du spectacle conserve une collection d'environ 500 masques de spectacles, soit réellement portés sur scène, soit des répliques jamais portées, ou encore des masques fabriqués à des fins pédagogiques ainsi que des modèles relevant de l'artisanat, collectionnés par des praticiens du spectacle. La numérisation n'est pas ici un acte de valorisation, mais un substitut à la consultation de l'original. Outre la vue d'ensemble, on tourne autour du masque dans le sens des aiguilles d'une montre pour capturer en huit prises de vues la totalité de l'objet auquel on ajoute, pour une information complète, une vue de dessus, une vue de dessous et une vue d'illustration de la technique de construction du masque. Deux éléments sont importants : le système d'attache et les trous pour les yeux. Il ne doit pas y avoir de vue spécifique pour cela : les vues circulaires doivent permettre de les voir. Contrairement au cas des marionnettes, la photographie d'un masque porté a été écartée du protocole, car sans plus-value et hors des limites de l'exercice documentaire.

\section{ANIMER L'OBJET : DES NUMÉRISATIONS COMPLEXES, DES INTERVENANTS D'APPUI}

\section{- Numériser en 3D, fantasme et expérimentation}

La 3D semble séduisante pour animer les objets. On envisage des techniques de modélisation 3D via la photogrammétrie, ou bien la triangulation laser (un plateau tournant sur lequel est posé l'objet, analysé par une caméra et un faisceau laser). Des exemples convaincants ont été présentés par le CNRS aux $7^{\mathrm{e}}$ journées de la conservation-restauration par exemple, mais très vite se posent des questions sur l'intérêt réel de cette technique, ses limites, sa plusvalue par rapport à une prise de vues $2 \mathrm{D}$ complète d'un point de vue documentaire. La $\mathrm{BnF}$ a ainsi bénéficié d'un mécénat de compétences de Dai Nippon Printing en 2015 pour numériser 55 globes : jusqu'à 600 images par globe, avec une modélisation réalisée à Tokyo, dans le cadre du projet «DNP Museum Lab ». Mais il a fallu séparer les globes de leurs piètements, et il a donc été nécessaire de compléter par une campagne de prises de vues $2 \mathrm{D}$ pour recontextualiser les images $3 \mathrm{D}$ et les raccrocher à l'objet physique complet. La diffusion sur Gallica impose des développements spécifiques et difficiles à maintenir. La pérennité de l'environnement logiciel reste aujourd'hui un point de faiblesse, et la 3D une action de valorisation non exclusive d'autres méthodes, comme la vidéo qui permet, elle, de capter le mouvement de l'objet. C'est pourquoi nos principaux chantiers restent aujourd'hui basés sur des prises de vue $2 \mathrm{D}$, dont les protocoles vont simuler les apports de la 3D tout en offrant une meilleure qualité d'image.

\section{Sublimer les costumes}

7000 costumes, environ 15000 pièces, conservés au département des Arts du spectacle, témoignent des costumiers comme des ateliers et portent en eux l'empreinte des interprètes. La prise de vue nécessite une mise en forme sur mannequin, et la $\mathrm{BnF}$ emploie à cet effet une costumière assistée de magasiniers, alliant connaissances techniques et connaissances de l'histoire du costume. Elle emploie des matériaux chimiquement neutres (ouates de polyester, toile naturelle, jersey de coton, tulle, crin et épingles de conservation...) pour étoffer la silhouette du mannequin, la doter de bras ou de jambes le cas échéant. La documentation iconographique est précieuse pour restituer l'univers esthétique d'origine. Un plateau tournant est indispensable à la réalisation de l'ensemble des vues circulaires. Ce dispositif permet une rotation fluide par quart de tour, sans avoir à manipuler le mannequin, ni à réajuster les tissus et les plis.

\section{Animer les marionnettes}

Le département des Arts du spectacle conserve aussi près de mille marionnettes : à fils, à gaine, marottes, ombres... Un quart d'entre elles est d'ores et déjà accessible dans Gallica et les prises de vue, qui donnent une vision très complète des objets, de leur fabrication et de leur technique de manipulation, se poursuivent. Il ne s'agit pas de refaire des photographies de spectacles avec mise en scène, mais de représenter les objets en rendant compte de leur technique de manipulation et de construction. La diversité d'aspect, de taille et de 


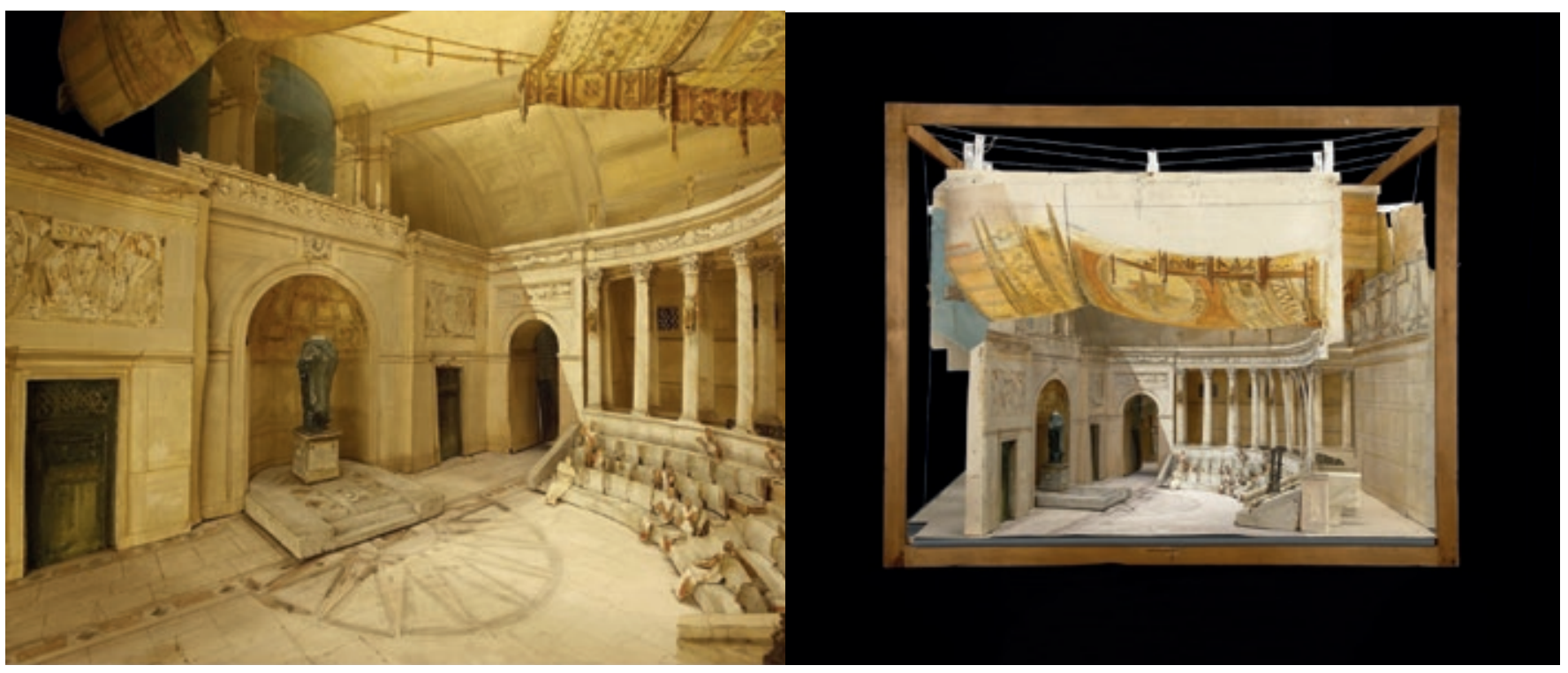

Maquette pour l'opéra Roma de Jules Massenet : maquette construite de l'acte IV par Alexandre Bailly (1866-1947) (coll. BnF)

fonctionnement nécessite parfois la présence de plusieurs personnes pour les manipuler. Dans certain cas (marionnettes à taille humaine, marionnettes à tiges nécessitant quatre mains...), les intervenants visibles sur les clichés sont vêtus de noir, selon un principe de neutralité et de discrétion. La manipulation des marionnettes est en effet une activité complexe et physique, qui nécessite du personnel disponible et volontaire.

\section{a collection Charles Cros : machineries en abymes}

Dans ce dernier cas, les prises de vues doivent rendre compte tant du dispositif général, du fonctionnement de l'appareil, du ou des usages, que des écarts entre l'appareil conservé et son modèle industriel. Les vues couvrent l'objet dans sa globalité, en fonctionnement, et ses accessoires. Les appareils doivent être rendus tant dans leur mode de lecture de son par exemple, que dans leur fonction de captation de la voix ou de l'image. La manipulation suppose beaucoup de manutention, aussi la présence des chargés de collection est-elle indispensable compte tenu de son aspect particulièrement technique. Prenons le cas du « puits à pétrole " (phonographe à contrepoids pour enregistrement professionnel, réalisé par Pathé et reproduit p. 23) : cet appareil se compose de trois parties qui, assemblées, mesurent plus de deux mètres. Lors de la prise de vue, le studio installé dans les réserves ne disposait pas de cette hauteur. L'appareil a été photographié en deux fois, puis les images assemblées sous Photoshop. Ce procédé a été réalisé cinq fois afin d'obtenir plusieurs points de vues. Compte tenu du très grand format, des gros plans ont été réalisés afin de mettre en valeur les vues techniques.

Pour mener à bien sa politique de numérisation des objets, la $\mathrm{BnF}$ associe pragmatiquement priorités documentaires et solutions techniques adaptées. La prise de vue classique reste à ce jour le meilleur compromis, grâce à la finesse des protocoles définis et au travail de mise en acte. Diversifier les solutions, via la vidéo, les scanners 3D ou d'autres technologies encore est notre nouveau chantier, qui nous confronte à deux défis : celui des moyens déjà considérables mobilisés par ces programmes, et celui de la maîtrise et de la pérennité des technologies de diffusion, dans le cadre de Gallica.

\section{À VOIR}

\section{Généralités}

- https://gallica.bnf.fr/html/und/cartes/les-globes-en-3d

- https://gallica.bnf.fr/html/und/objets/collection-charles-cros

- https://gallica.bnf.fr/html/und/objets/monnaies

- https://gallica.bnf.fr/html/und/objets/collections-de-marionnettes-titre-page

- https://gallica.bnf.fr/html/und/objets/collections-demasques-titre-page 